Atmos. Chem. Phys., 10, 2475-2490, 2010

www.atmos-chem-phys.net/10/2475/2010/

(C) Author(s) 2010. This work is distributed under

the Creative Commons Attribution 3.0 License.

\title{
Linking urban aerosol fluxes in street canyons to larger scale emissions
}

\author{
B. K. Tay ${ }^{1}$, G. B. McFiggans ${ }^{1}$, D. P. Jones ${ }^{2}$, M. W. Gallagher ${ }^{1}$, C. Martin $^{1}$, P. Watkins ${ }^{2}$, and R. M. Harrison ${ }^{3}$ \\ ${ }^{1}$ Centre for Atmospheric Science, SEAES, The University of Manchester, Manchester, M13 9PL, UK \\ ${ }^{2}$ School of Mechanical, Aerospace and Civil Engineering, The University of Manchester, P.O. Box 88, \\ Manchester M60 1QD, UK \\ ${ }^{3}$ Division of Environmental Health \& Risk Management, School of Geography, Earth \& Environmental Sciences, University \\ of Birmingham, Edgbaston, Birmingham B15 2TT, UK
}

Received: 12 August 2009 - Published in Atmos. Chem. Phys. Discuss.: 1 September 2009

Revised: 14 January 2010 - Accepted: 2 March 2010 - Published: 11 March 2010

\begin{abstract}
In this study we investigate ultrafine particle (UFP) fluxes using a first order eddy viscosity turbulence closure Computational Fluid Dynamics (CFD) model and determine the different factors that influence emissions of UFP into the urban boundary layer. Both vertical turbulent fluxes as well as the fluxes due to mean circulatory flow are shown to contribute to the overall ventilation characteristics of street canyons. We then derive a simple parameterised numerical prediction model for canyon top UFP venting which is then compared with tower based micrometeorological flux measurements obtained during the REPARTEE \& CityFlux field experiments.
\end{abstract}

\section{Introduction}

The spatial heterogeneity of urban street canyons and the complex interplay of chemically, spatially and temporally varying ultrafine particle (UFP) emission sources as a function of micrometeorological and meteorological factors represents a challenge to both dispersion modelling activities and field observations. Measurement campaigns generally have insufficient resolution to represent aerosol particle transport and transformation adequately and must be informed by appropriate dispersion modelling techniques.

In this study we investigate UFP fluxes using a first order eddy viscosity turbulence closure Computational Fluid Dynamics (CFD) model to determine the different factors that

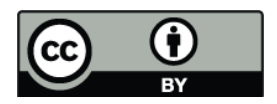

Correspondence to: M. W. Gallagher (martin.gallagher@manchester.ac.uk) influence the UFP vertical fluxes from a street canyon. Both turbulent fluxes and fluxes due to mean flows are shown to contribute to the overall ventilation characteristics of a street canyon. We then derive a simple parameterised numerical prediction model for UFP venting at the roof level of the canyon. This simple model is then compared with tower based micrometeorological flux measurements reported in recently published field studies. Whilst undoubtedly crude these comparisons may be used as a starting point for linking street level concentrations to those measured above the urban roughness sublayer with potential for validating high resolution neighbourhood-scale air quality models.

\section{Background}

An understanding of the ventilation characteristics of street canyons under a range of flow conditions will aid the prediction of the average UFP pollutant concentrations for street level pollutant exposure assessment, pollutant monitoring network design and data interpretation. It is also the first step in coupling street level UFP concentrations to net emission fluxes measured above the urban roughness sublayer, for assessing regional and climate model impact and mitigation studies.

Implicit in most operational street canyon models is that the canyon is venting vertically. Whilst it is understood that vertical fluxes from street canyons are governed by both turbulence and advection in the vertical direction, due to complex circulatory flows within the canyon, the relative importance of each process is a topic of ongoing research. Advection originates from the mean flow of roof level wind

Published by Copernicus Publications on behalf of the European Geosciences Union. 
conditions and turbulence arises from unsteady flows caused by eddy motions in the air. The sources of turbulence are buoyancy, shear at the surfaces, traffic movement and the turbulent intensity of the flow above the canyon. If we consider the case of wind blowing perpendicular to a canyon axis, the layer of strong shear that develops at the canyon top is believed to oscillate, driving an intermittent mixing circulation around the street canyon (Belcher, 2005). Pollutants travelling up the leeward face of a canyon require sufficient momentum to penetrate the shear layer and be transported into the overlying boundary layer and this takes place over relatively long timescales ( 30-60 s) (Walton and Cheng, 2002). Using a 2-D Computational Fluid Dynamics (CFD) model coupled with the standard $k-\varepsilon$ turbulence model and considering wind perpendicular to the longitudinal axis of an idealised canyon, (e.g. aspect ratio $H / W \sim 0.5-2$ where $H$ is the canyon height and $W$ its width; Turbulent Intensity $\sim 0.001-$ 0.025 and wind speeds $\leq 5 \mathrm{~m} / \mathrm{s}$ ) it has been shown that the turbulent flux contribution dominates the net vertical flux of pollutants as it was found to be an order of magnitude larger than the advective flux. In addition, the net effect of turbulent flux has been found to exhaust pollutants whilst the net effect of advective flux is to re-entrain pollutants (Baik and Kim, 2002).

There is evidence from past experimental studies that canyon ventilation is a function of a range of flow conditions, in particular turbulence, wind speed and canyon geometry. In a field study, DePaul and Sheih (1986) found that the ventilation velocity of a tracer from a canyon with aspect ratio $H / W=1.5$ was correlated with both friction velocity, $u_{*}$, at the roof level, and the horizontal wind, $U$. In addition, Barlow and Belcher (2002) found that the street canyon aspect ratio is an important factor in influencing the ventilation efficiency. It has also been found that amongst all flow regimes, ventilation efficiency is most dominant for wake-interference flow. Both studies have found a robust relationship between mean ventilation velocity and horizontal wind component, suggesting that the scalar transport is controlled by turbulence (Barlow and Belcher, 2002). This hypothesis is consistent with the numerical studies referred to above.

In spite of extensive studies on the escape of pollutants from street canyons, there are still several research questions warranting further investigation. There has been little attempt to parameterise flux from street canyons at a range of turbulent intensities, wind speeds and canyon aspect ratios. The coupling of surface fluxes and dynamics to neighbourhood scale fluxes for large-scale models, within the urban canopy, though plausible, has also received little attention. Finally, whilst the dominance of turbulent flux under conditions where only forced convection from the wind has been demonstrated, previous studies have suggested that the velocity flow pattern within the canyon varies with solar angle (i.e. time of day) (e.g. Nakaruma and Oke, 1988) due to natural convection arising from thermal effects within the canyon. The influence of mixed convection on the relative extents of both turbulent and vertical advective fluxes at roof level has not been studied in detail and needs further investigation.

\section{CFD model framework and results}

For this study an in-house two dimensional (2D) CFD modelling platform based on the incompressible finite volume method (Patankar, 1980) was used. The mass, momentum and standard $k-\varepsilon$ turbulence model (Launder and Spalding, 1974) equations representing the continuous phase were solved. An additional energy equation was solved when considering buoyancy cases based on the Boussinesq Approximation. The discrete phase, representing UFP, was characterised via the Modal Method (Whitby et al., 1991) and assumed to be transported by the velocity field for the continuous phase. Due to the low volumetric loading and stokes number of UFP, this assumption is a plausible one. The equations were solved using the deferred correction Total Variation Differencing Scheme except for the turbulence equations, which were solved using the Upward Differencing Scheme. The SIMPLE (Semi Implicit Method for Pressure Linked Equations) pressure-velocity coupling scheme was used to obtain the velocity field. Details may be found in Ferziger and Peric (1999) and Versteeg and Malalasekera (2007). Steady-state solutions were obtained for all cases.

The code was validated against a benchmark 2-D cavity test case (Ketzel et al., 2004). The solution of the code was compared with wind tunnel databases to assess the model skill in solving the mass, momentum, turbulence (standard $k$ $\varepsilon$ turbulence model) equations. The flow obtained within the cavity by the model is characterised by a main re-circulation vortex and a secondary vortex at the leeward side of the cavity close to the ground (Fig. 1a). This result is quantitatively consistent with both wind tunnel databases (Fig. 2) (Ketzel et al., 2004) and previous numerical simulation (Fig. 1b) (Savory et al., 2004) of the same case. The horizontal velocity profiles obtained using the model of this study along 3 axes within the cavity compares well with wind tunnel database (Fig. 2a, b and c).

The computational domain comprised of the symmetry, inlet and outlet boundary conditions with a cavity below representing an idealized street canyon (Fig. 3). The height of the canyon $(\mathrm{H})$ was chosen to be $10 \mathrm{~m}$, a typical length scale expected in urban environments. Smooth wall boundary conditions were assumed. The inlet and outlet boundary conditions were $2 \mathrm{H}$ and $10 \mathrm{H}$ away from the canyon. The symmetry boundary condition was $5 \mathrm{H}$ above the canyon. Boundary conditions were located at a distance such that they would not interfere with the numerical results within the cavity. A structured mesh was used and a total of 70, 500 grid cells were used to model dispersion in a $10 \mathrm{~m}$ by $10 \mathrm{~m}$ canyon.

Appropriate values representing a range of meteorological conditions were used at the inlet boundary conditions where a uniform wind speed profile $(U)$ was imposed representing 

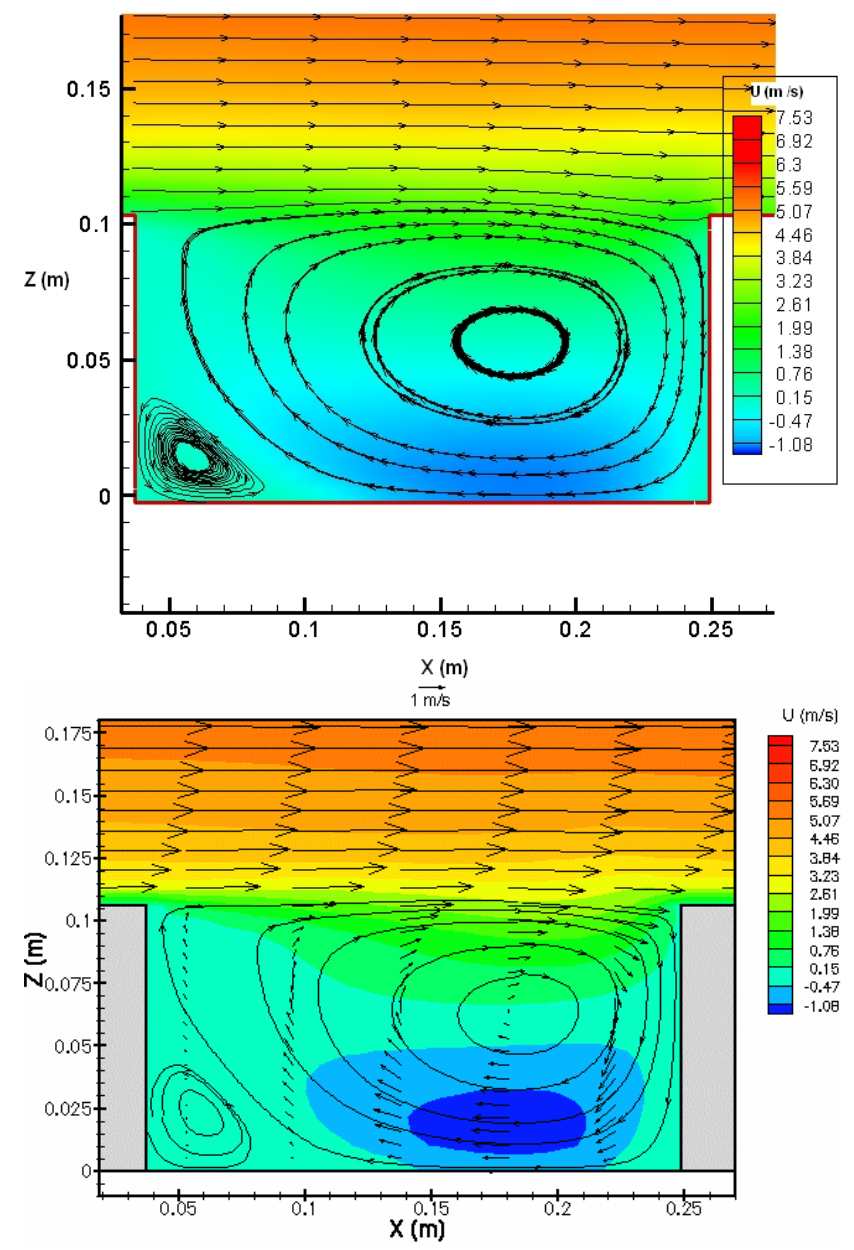

Fig. 1. (a) Numerical results (b) Previously published model results of the same case (Savory, et al., 2004).

wind blowing perpendicular to the canyon axis. Although a uniform wind speed was incorporated at the inlet boundary condition, the location of the inlet boundary condition 2 $\mathrm{H}$ from the canyon allows a logarithmic wind profile to develop due to velocity shear at the roof. It is acknowledged that this method of characterisation is idealised and only adopted for the purposes of sensitivity studies. The range of wind speeds $(2.5 \mathrm{~m} / \mathrm{s}$ to $10 \mathrm{~m} / \mathrm{s})$ selected represented the flow regime where the extent of forced convection is such that vehicular turbulence could be ignored (Kumar, et al., 2009 and Longley, 2004).

The turbulent kinetic energy profile at the inlet boundary condition was set equal to:

$k=1.5 \times T I^{2} \times U^{2}$

Values of TI (TI, the ratio of the root-mean-square of the velocity fluctuations, $u$, to the mean flow velocity) chosen were $0.26,0.1$ and 0.05 , representing 3 orders of magnitude of turbulence intensities, well within the range of TI commonly
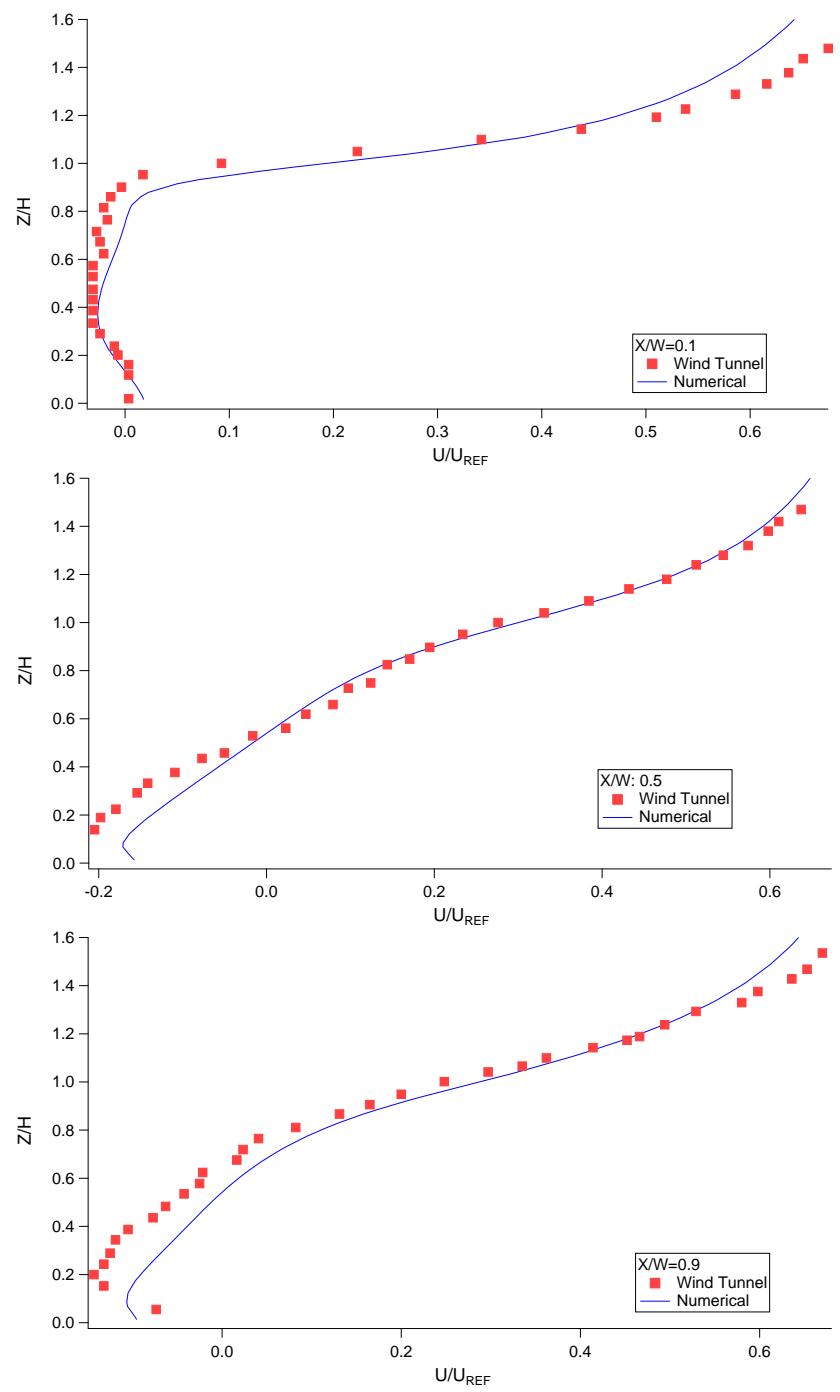

Fig. 2. Comparison of numerically computated wind profile using the model of this study with wind tunnel databases (Ketzel et al. , 2004) at (a) $\mathrm{X} / \mathrm{W}=0.1$; (b) 0.5 and (c) 0.9

used for such modelling studies (Sini et al., 1996; Baik and Kim, 1999, 2002, 2003; Jeong and Andrew, 2002; Solazzo and Britter, 2007; Kumar et al., 2009; Murena and Favale, 2009) and to allow the investigation of the effects of H/W on ventilation characteristics. Higher levels of turbulence intensity will conceal geometrical influences and thus hinder our investigation (Sini et al., 1996)

The inlet turbulent dissipation profile was set to:

$\varepsilon=C_{\mu}^{0.75} k^{1.5} z^{-1}$

with $C_{\mu}=0.09$ and the length scale $(z)$ was taken to be 0.07 of the characteristic length scale of the velocity inlet (Versteeg and Malalasekera, 2007).

To represent vehicle exhaust plumes, an elevated finite cross sectional line "emission source" $0.3 \mathrm{~m}$ above ground 


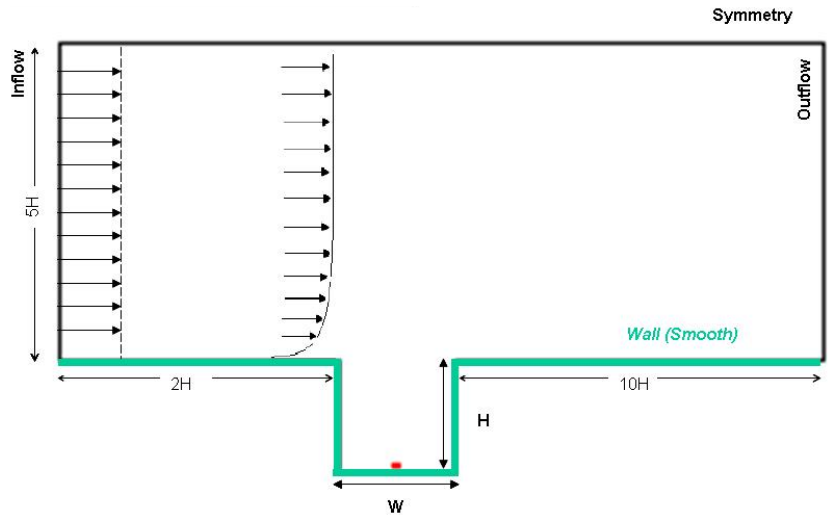

Fig. 3. Computational domain.

was imposed with a predetermined concentration level of the discrete phase (Table 1). It was based on an a-priori assumption of a typical Aitken and accumulation aerosol particle lognormal size distribution for representative concentrations $1 \mathrm{~m}$ from a vehicle exhaust pipe (Kittelson, 1998). The characteristisation of particle size distribution features will allow for future investigation into aerosol processes in subsequent studies. The dilute nature of UFP is consistent with the oneway coupling assumption. The background UFP concentration was assumed to be zero. The turbulent Schmidt number was set to 1 (Kumar et al., 2009). The "zero concentration" wall boundary condition (perfectly absorbing wall) was used for UFP, ignoring re-entrainment. It is a "good approximation for commonly encountered aerosols in ambient air" (Gallis et al., 2008), although this simplified estimate may overestimate deposition flux.

\subsection{Flux characterisation and parameter space}

\subsubsection{Characterisation of turbulent and vertical advec- tive flux components}

In this study both turbulent and vertical advective fluxes were evaluated at the roof level of the canyon by integration across the width of the canyon cavity, which is the interface of the canyon and free-flow regime above. A positive flux implies a net venting from the canyon while negative flux implies a net re-entry into the canyon. The vertical UFP advective flux due to mean flow is: $f_{a}=\overline{w \chi}$; where $\bar{\chi}$ is the mean UFP concentration and $\bar{w}$ is the mean vertical velocity at canyon roof level. Implementing a first order eddy viscosity turbulence closure model, the turbulent flux may be characterised as the product of the turbulent diffusivity and the vertical gradient of UFP concentration. The vertical flux of UFP due to turbulent flow is therefore: $f_{t}=w^{\prime} \chi^{\prime}=-K_{\chi} \frac{\partial \chi}{\partial z}$ where $\chi^{\prime}$ is the deviation from the mean concentration and $w^{\prime}$ is the deviation from the mean vertical velocity, $\mathrm{z}$ is height in the vertical direction and $K_{\chi}$ is the turbulent diffusivity of UFP. Integration of the flux along the horizontal direction yields the UFP
Table 1. Aerosol particle size distributions assumed at the emission point.

\begin{tabular}{lll}
\hline & $\begin{array}{l}\text { Aitken } \\
\text { Mode }\end{array}$ & $\begin{array}{l}\text { Accumulation } \\
\text { Mode }\end{array}$ \\
\hline Number Concentration (particles $\left./ \mathrm{m}^{3}\right)$ & $1 \times 10^{11}$ & $1 \times 10^{10}$ \\
Geometric Mean Diameter $(\mathrm{nm})$ & 15 & 150 \\
Standard Deviation & 1.5 & 1.6 \\
Volume Fraction $\left(\mathrm{m}^{3} / \mathrm{m}^{3}\right)$ & & $4.5 \times 10^{-11}$ \\
\hline
\end{tabular}

Table 2. Model canyon and flow characteristics at inflow boundary condition.

\begin{tabular}{llll}
\hline & Low & Medium & High \\
\hline Aspect Ratio $(H / W)$ & 0.50 & 1.0 & 2.00 \\
Turbulence Intensity $(T I)$ & 0.05 & 0.1 & 0.26 \\
Wind Speed $U(\mathrm{~m} / \mathrm{s})$ & 2.50 & 5.0 & 10.00 \\
\hline
\end{tabular}

flux from the canyon due to vertical advection, $F_{a}=\int_{W} f_{a} d x$, and turbulent flow $F_{t}=\int_{W} f_{t} d x$, where $W$ is the width of the canyon. The net UFP flux from both processes along the horizontal axis of the canyon at roof level is thus expressed as:

$F_{\text {Net }, \chi}=\int_{W}\left(-K_{\chi} \frac{\partial \chi}{\partial z}+\overline{\chi w}\right) d x$

Similarly, for mixed convection cases, local heat flux was estimated at the roof of the canyon and expressed as:

$F_{\mathrm{Net}, T}=\int_{W}\left(-K_{T} \frac{\partial T}{\partial z}+\bar{T} \bar{w}\right) d x$

where $T$ is temperature and $K_{\mathrm{T}}$ is the turbulent diffusivity of heat.

\subsubsection{Parameter space: isothermal cases}

Natural buoyancy effects due to heated walls and exhaust plumes were ignored in these cases. Table 2 summarises the parameter space for the studies where TI and inflow levels are referred to hereafter as low, medium and high cases. The Reynolds Number $\operatorname{Re}=\frac{U \rho H}{\mu}$ (where $\rho$ is the air density, $\mu$ the viscosity, and $U$, the canyon inflow wind speed measured at a reference height, and $H$ is again the canyon height) is the ratio of the inertial and viscous forces and range from $1.7 \times 10^{6}$ to $6.7 \times 10^{6}$.

\subsubsection{Parameter space: mixed convection cases}

To investigate the influence of thermal effects within canyons on the UFP flux, a canyon of unity aspect ratio was 
Table 3a. Leeward heated wall conditions.

\begin{tabular}{|c|c|c|c|c|c|c|c|c|}
\hline Case & 1 & 2 & 3 & 4 & 5 & 6 & 7 & 8 \\
\hline$U(\mathrm{~m} / \mathrm{s})$ & & 10 & & & & 5 & & \\
\hline$\Delta T(\mathrm{~K})$ & 4 & 10 & 15 & 2 & 4 & 6 & 10 & 15 \\
\hline$R_{i}$ & 0.0135 & 0.0338 & 0.0507 & 0.0271 & 0.0541 & 0.0812 & 0.1353 & 0.2030 \\
\hline
\end{tabular}

Table 3b. Leeward heated wall conditions.

\begin{tabular}{llllll}
\hline Case & 9 & 10 & 11 & 12 & 13 \\
\hline$U(\mathrm{~m} / \mathrm{s})$ & & \multicolumn{5}{c}{2.5} \\
$\Delta T(\mathrm{~K})$ & 4 & 6 & 8 & 10 & 15 \\
$R_{i}$ & 0.217 & 0.325 & 0.433 & 0.541 & 0.812 \\
\hline
\end{tabular}

considered and the temperature of the UFP source exhaust approximately $1 \mathrm{~m}$ away from the exhaust pipe was assumed to be $300 \mathrm{~K}$, whilst air temperature above the canopy was $290 \mathrm{~K}$. The highest $T I$ value (0.26) was assumed for these cases. Either the leeward or windward wall was assumed to be heated. The heated wall was assumed to have a temperature higher than the ambient $(290 \mathrm{~K})$. The relative thermal effects of buoyancy and forced convection within a street canyon may be determined based on the Richardson Number, $R_{i}=\frac{g H \Delta T}{U^{2} T_{0}}$, the ratio of potential to kinetic energy where $g$ is the gravitational constant, $\Delta T$ is the temperature difference between the heated wall and the above canyon flow, and $T_{0}$ is the above-canyon air temperature. At low $R_{i}$, the temperature difference between the heated wall and the fluid is small and the wind speed large enough so that buoyancy effects may be ignored, but beyond a critical value, buoyancy becomes important enough to affect the overall fluid flow pattern. Tables $3 \mathrm{a}-\mathrm{c}$ summarises the ranges of parameter space tested.

\subsection{CFD model results: isothermal cases}

\subsubsection{Isothermal cases: velocity flow patterns}

Skimming flow was observed for all cases considered, characterized by a main clockwise vortex extending throughout the canyon geometry and 3 minor anti-clockwise vortices at the two corners of the leeward side as well as the bottom corner of the windward side, Fig. 4. Compared with other geometries $(H / W=0.5$ and 2.0$)$, the corner vortices are more dominant at $H / W=1.0$. The centre of the vortex appeared in the middle of the canyon when the aspect ratio $(H / W)$ was 1.0 and 2.0 , displacing to the windward side when the aspect ratio was 0.5 (Fig. 4c). This agrees with Johnson and Hunter's (1999) observations of a skimming flow regime down to an aspect ratio of 0.4 (be- low which wake-interference flow takes over) and Solazzo and Britter's (2007) numerical simulations, assuming smooth wall boundary conditions. This contrasts with other studies where a dual-vortex skimming flow regime was observed at $H / W \sim 2.0$ when rough wall conditions were considered, and a transitional threshold to the wake interference flow observed at $H / W \sim 0.65$ (e.g. Hunter et al., 1992 and Sini et al., 1996). Due to this vortex flow, advection at roof level was found to contain both the updraft and downdraft contributions at the leeward and windward side of the canyon respectively. The relative contribution of either component determines the net direction of vertical advective flux, as will be discussed in the following section.

\subsubsection{Isothermal cases: concentration profiles}

Before discussing in detail the results for UFP fluxes we note in passing that previous CFD experiments (Kumar et al., 2009) have shown varying degrees of success in representing real-world UFP concentration profiles measured in street canyons. We attempt to compare the concentration profiles that we have obtained for this study with previous field measurements of UFP concentrations (Kumar et al., 2009) and wind tunnel measurements of passive scalar concentrations (Meroney et al., 1996).

Figure 6a is the leeward canyon vertical UFP concentration profile predicted using this model for for the $H / W: 1$ canyon, $U=2.5 \mathrm{~m} / \mathrm{s}$ and $T I$ : 0.1 . The predicted profile structure can be split into three general layers; a layer near street level where concentrations increase rapidly to a maximum value, a middle layer where the concentration follows an approximate exponential decrease and a turbulent shear layer at the top of the canyon where the concentration declines more rapidly. The predicted leeward profile appears to be in reasonably qualitative agreement with the field measurements of Kumar et al. (2009), also shown in Fig. 6a, despite the simplifications, although such measurements we note are still sparse. Fig. 6b also shows the windward concentration profile that has different characteristics and which should be considered in the design and interpretation of canyon field experiments.

An attempt was made to compare vertical concentration profile trends for this study and those obtained from (Meroney et al., 1996) (Fig 6c). For all simulation cases in this study, an elevated exhaust $(0.3 \mathrm{~m})$ was considered as 
Table 3c. Windward heated wall.

\begin{tabular}{llllllllll}
\hline Case & 1 & 2 & 3 & 4 & 5 & 6 & 7 & 8 & 9 \\
\hline$U(\mathrm{~m} / \mathrm{s})$ & & 10 & & \multicolumn{2}{c}{5} \\
$\Delta T(\mathrm{~K})$ & 4 & 10 & 15 & 4 & 10 & 15 & 4 & 10 & 15 \\
$R_{i}$ & 0.0135 & 0.0338 & 0.0507 & 0.0541 & 0.1353 & 0.2030 & 0.2165 & 0.5412 & 0.8119 \\
\hline
\end{tabular}

well as the fully absorbing wall, contrary to the wind tunnel study where the source was at ground level and deposition flux was assumed to be zero. For both studies, (Fig. 6a and c), a higher concentration was observed at the leeward side, consistent with the clockwise vortex flow. A sharp concentration gradient within the "turbulent shear layer" near the roof was also observed for both cases. Some qualitative discrepancies were observed: the windward structure observed by Meroney et al. (1996) was homogenous, but a convex structure was observed for this study and the leeward structure of this study observed a positive gradient near the ground, which is different for the Meroney et al. (1996) structure where the ground level exhibited the maximum. These discrepancies could be accounted for by the aerosol particle deposition process and the elevated source considered within the model adopted for this work, though further investigation is needed to refine the characterisation of deposition in this model and to fully account for the differences between particulate and gaseous behaviour.

\subsubsection{Isothermal cases: UFP fluxes}

Consistent with Baik and Kim's (2002) studies, for all cases, $F_{\mathrm{Net}, \chi}$ was found to be positive, implying a net venting of UFP into the urban boundary layer. A positive $F_{t}$ (venting of UFP), was observed for all $H / W$ (Fig. 7). The greater magnitude of turbulent flux at lower $H / W$ suggests better ventilation characteristics. $F_{a}$ is positive when $H / W=0.5$ but negative (re-entrainment of UFP) for the other geometries (Fig. 10). The magnitude of turbulent flux, $F_{t}$ is higher than the vertical advective flux, $F_{a}$ by at least an order of magnitude for all cases, consistent with the data reported by Baik and $\mathrm{Kim}(2002)$, except for shallow canyons $(H / W=0.5)$ at $10 \mathrm{~m} / \mathrm{s}$ when the turbulent flux is higher by 2 orders of magnitude. When considering forced convection alone and when effects of buoyancy may be ignored, the turbulent flux dominates the ventilation process. This is consistent with the suggestion of Barlow and Belcher (2002).

For a given wind speed, an increase in $T I$ will result in a proportional increase in $F_{t}$. For all cases considered, there is an increase in $F_{t}$ with increasing $T I$ as expected for a given $U$, Fig. 7. It was observed that the sensitivity of $F_{t}$ to $T I$ is dependent on $H / W$, being strongest for the symmetrical $(H / W=1.0)$ canyon. The reasons for the weaker sensitivity for $H / W=0.5$ and 2 are different. $F_{t}$ is a function of tur- bulent diffusivity $\left(K_{\chi}\right)$, and vertical concentration gradient, $d \chi / d z$. For all cases, the increase in $T I$ increases the mean $K_{\chi}$. When $T I$ increases from 0.05 to 0.1 , the overall vertical concentration gradient may increase (due to enhanced vertical advection) but, when $T I$ increases from 0.1 to 0.26 , the vertical concentration gradient may decrease due to the enhanced turbulent mixing of pollutants.

These trends are summarised in Fig. 8, for the case $U=10 \mathrm{~m} / \mathrm{s}$, which shows the vertical concentration gradient $(d \chi / d z)$ evaluated at the roof level along the cavity width as a function of normalised distance, $X / W$ (centre of canyon zero, leeward wall -1 and windward wall +1 ), across the canyon, and for the different canyon aspect ratios. Observing both the $H / W=0.5$ and $H / W=2.0$, the maximum vertical concentration gradient occurs just next to the leeward side of the canyon, but for $H / W=1$, it is further away from the wall. When $H / W=0.5$, enhanced mixing due to increased turbulence leads to a reduction of vertical concentration gradient along the entire horizontal axis, which moderates the increase in $F_{t}$ due to enhancement in eddy viscosity when $T I$ increases. In contrast, when $H / W=2.0$, the decrease in vertical concentration gradient was found to be less apparent and least sensitive to $T I$, taking place only at the windward side of the canyon. This implies minimal advection of turbulent quantities into deeper canyons and consequently the weakest sensitivity to $T I . F_{t}$ for the $H / W=1.0$ canyon, however, is the most sensitive to $T I$. This is because whilst there is a reduction in vertical concentration gradient at the windward side of the canyon due to turbulent mixing, there is also a corresponding enhancement of concentration gradient at the leeward side in this case. This is not observed for the other two canyon geometries and therefore accounts for the greatest sensitivity of $H / W=1.0$.

\subsubsection{Isothermal cases: turbulent and vertical advective UFP flux}

For a given $T I$, an increase in $U$ will result in a proportional increase in $F_{t}$, Fig. 9. This strong relation is consistent with observations by Barlow and Belcher (2002) and DePaul and Sheih (1986). This is because the increase in $U$ enhances the eddy viscosity and vertical concentration gradient at the roof of the canyon.

At all $H / W$ values for a given $T I$, the magnitude of $F_{a}$ increases as the inflow $U$ increases, Fig 10. This is because the 

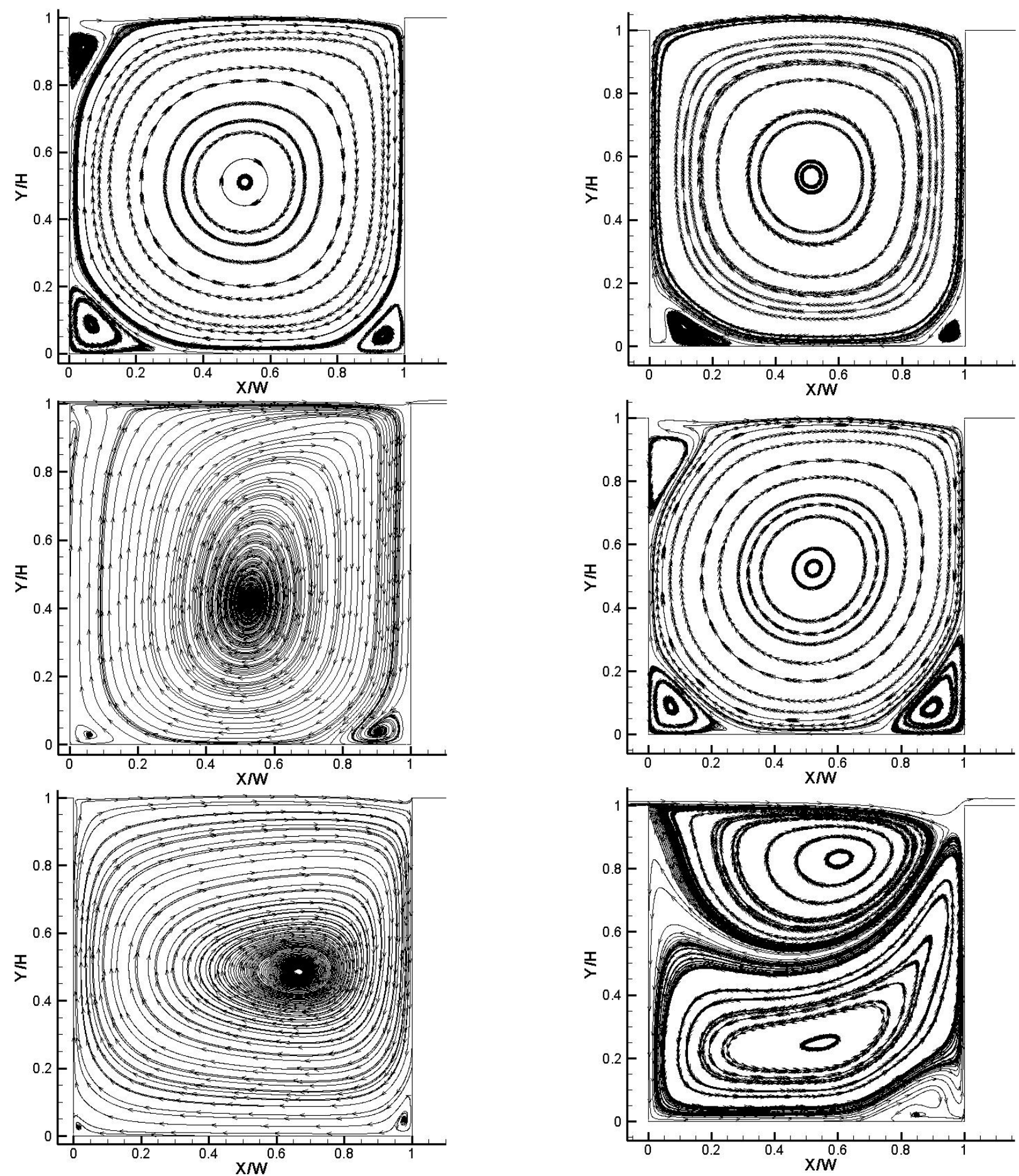

Fig. 4. Typical streamline patterns produced by the model for various canyon aspect ratios $(H / W)$ : (a) $H / W=1.0$; (b) $H / W=2.0$ and (c) $H / W=0.5$ (Isothermal case with $U=5 \mathrm{~m} / \mathrm{s}$ and medium level turbulence intensity (TI: 0.1$)$ ). Vertical axis is normalised canyon height, horizontal axis is normalised by canyon width.

Fig. 5. Typical streamline patterns produced by model for (a): leeward heated wall $(U=2.5 \mathrm{~m} / \mathrm{s}, \Delta T=15 \mathrm{~K}) ;(\mathbf{b})$ : for windward heated wall (mixed convection case for $U=2.5 \mathrm{~m} / \mathrm{s}, \Delta T=4 \mathrm{~K}$ ) and (c): windward heated wall showing dual-vortex flow (for $U=2.5 \mathrm{~m} / \mathrm{s}, \Delta T=15 \mathrm{~K}$ ). All cases for $H / W=1.0$ and $T I=0.26$. 
B. K. Tay et al.: Linking urban aerosol fluxes in street canyons
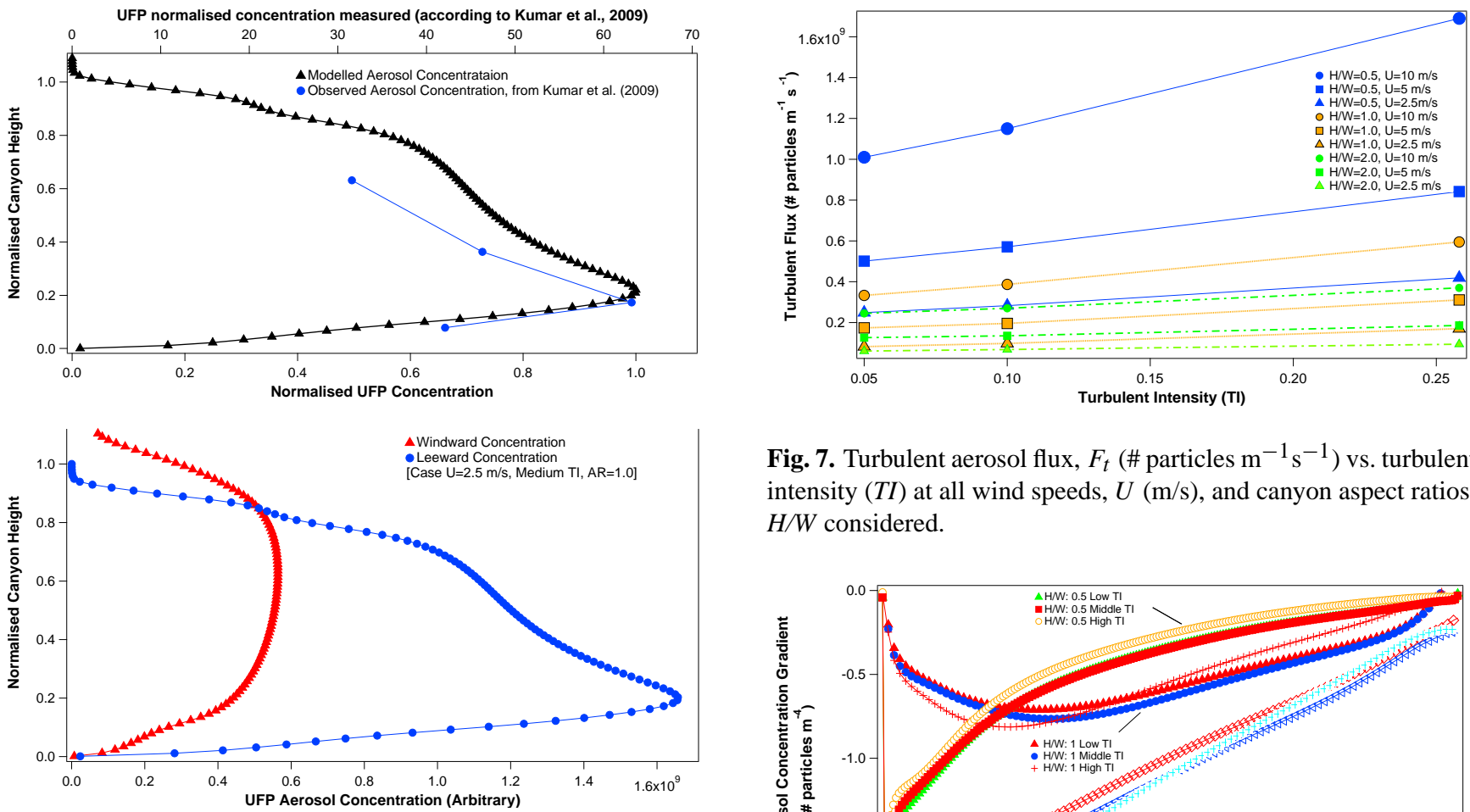

Fig. 7. Turbulent aerosol flux, $F_{t}\left(\#\right.$ particles $\left.\mathrm{m}^{-1} \mathrm{~s}^{-1}\right)$ vs. turbulent intensity $(T I)$ at all wind speeds, $U(\mathrm{~m} / \mathrm{s})$, and canyon aspect ratios, $H / W$ considered.

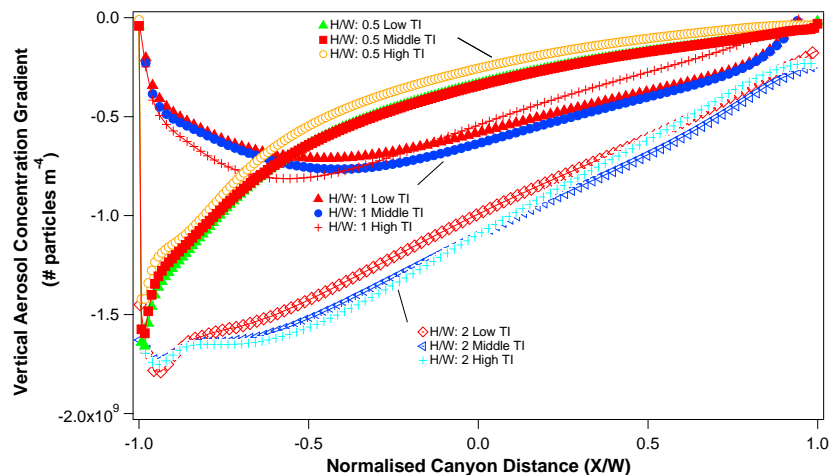

Fig. 8. Vertical concentration gradient profile: Vertical concentration gradient, vs. $X / W$, where $X$ is the distance across the canyon for $H / W: 0.5,1.0$ and 2.0, and low, medium and high turbulence intensities (TI) (see Table 2).

mean vertical velocity, $\bar{w}$ is in the positive direction across the majority of the axis at roof level, due to the displaced vortex centre. For a given $U$, an increase in $T I$ decreases the magnitude of $F_{a}$ for $H / W=1.0$ ( $F_{a}$ is negative). For $H / W=0.5$, when $F_{a}$ is positive for all cases, increases in $T I$ will result in an increase in $F_{a}$. This means that the effect of enhanced turbulence for both canyon geometries is an increased loss of UFP at the leeward canyon side. This trend is similar for both cases and reflects the strong interaction between in-canyon dynamics and the above-roof canopy flow where venting-in takes place at the windward side and venting-out, at the leeward side. In constrast, for canyon $H / W$ values $=2.0$ an increase in $T I$ increases $F_{a}$ (with increased entrainment). This reflects the weaker interaction between the canyon and the above-roof canopy flow, with one dominant advective direction at the windward side of the canyon.

vortex becomes more pronounced with increasing $U$.Further, observing Fig. 10, except for $H / W=0.5$, the net effect of vertical advection is toward entrainment of UFP into the canyon. The positive net contribution from the canyon into the urban "surface layer" by $F_{a}$ when $H / W=0.5$ occurs because the

\subsubsection{Isothermal cases: net UFP fluxes}

The net flux, $F_{\text {Net, } x}$ was then related to both $T I$ and $U$ for $H / W=0.5,1.0$ and 2.0 respectively. The exponential 


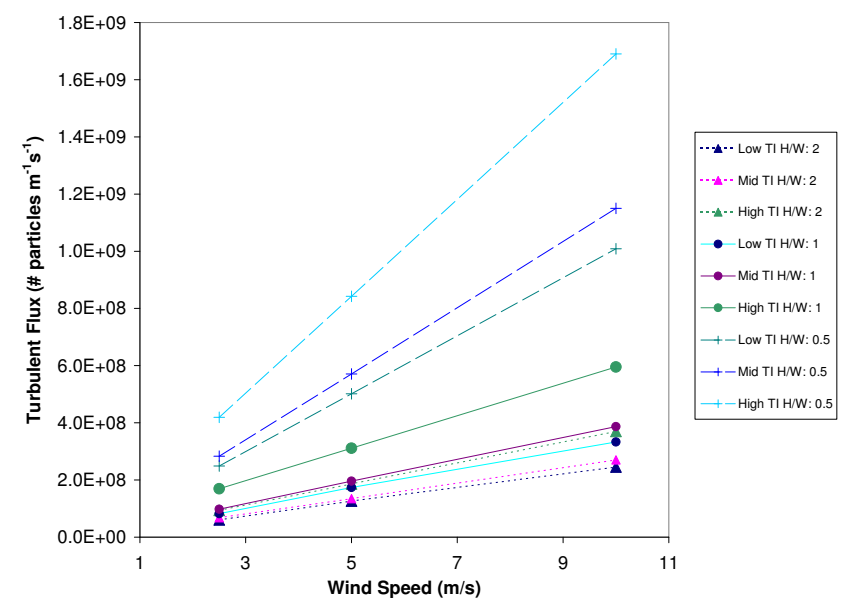

Fig. 9. Turbulent aerosol flux, $F_{t}$, vs. wind speed, $U(\mathrm{~m} / \mathrm{s})$ at all Turbulence Intensities, $T I$ and canyon aspect ratios, $H / W$ considered.

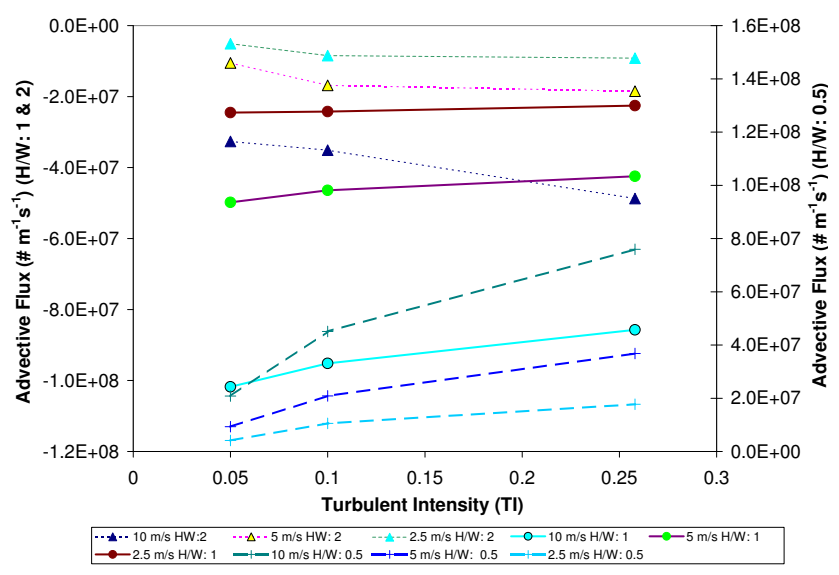

Fig. 10. Advective aerosol flux, $F_{a}$, vs. turbulent intensity, TI at all wind speeds, $U(\mathrm{~m} / \mathrm{s})$, and canyon aspect ratios, $H / W$ considered.

relationship observed between turbulence and $F_{\mathrm{Net}, \chi}$ and the linear relationship between $U$ and $F_{\mathrm{Net}, \chi}$ is characteristic of $F_{t}$. This demonstrates (as expected) the dominance of turbulence in the overall canyon venting process. A multi-variable regression was performed, using TableCurve $3-\mathrm{D}^{T M}$, on the data to relate the integrated net vertical flux from the canyon at roof level, $F_{\mathrm{Net}, \chi}$, to different levels of $T I$ and $U$. Several best-fit equations were proposed and solutions with $r^{2}$ of at least 0.99 were considered. The simplest result, which seems to best represent the process, took the following form;

$F_{\text {Net }, \chi}=\exp (a+b \ln U+c T I)$

where $a, b$ and $c$ are non-dimensional coefficients for a given source strength. This parameterisation applies for $H / W=0.5$, 1 and 2, single-vortex skimming flow regime and flow conditions assumed. Table 4 summarises the coefficients obtained in each case. Coefficient $b$ is similar for all aspect ratios,
Table 4. Model fit parameters.

\begin{tabular}{llll}
\hline Aspect Ratio & \multicolumn{2}{l}{$\begin{array}{l}\text { Coefficient values for } \\
\text { proposed parameterisation. }\end{array}$} \\
\hline & $a$ & $b$ & $c$ \\
0.5 & 18.31 & 1.01 & 2.55 \\
1 & 16.96 & 0.93 & 3.73 \\
2 & 16.88 & 0.96 & 1.99 \\
\hline
\end{tabular}

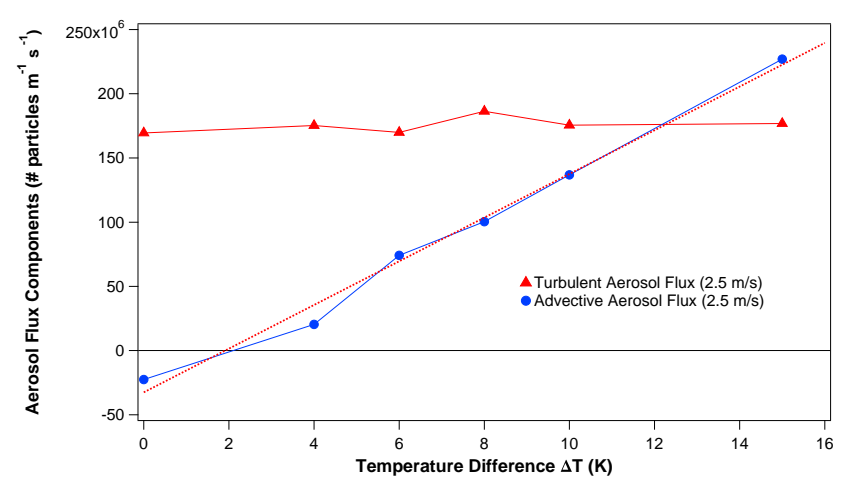

Fig. 11. Aerosol flux components $\left(F_{t}\right.$ and $\left.F_{a}\right)$ vs wall temperature difference $\Delta T(\mathrm{~K})$ at leeward wall for $U=2.5 \mathrm{~m} / \mathrm{s}$.

but a difference is observed for coefficient $c$, reflecting the different responses of flux to TI, discussed in Sect. 3.2.3.

\subsection{CFD model results: mixed convection cases}

\subsubsection{Mixed convection cases: velocity flow patterns}

Figure 5a shows an example of flow streamlines produced for a canyon with $H / W=1.0$ (leeward canyon wall heated, low wind speed $U=2.5 \mathrm{~m} / \mathrm{s}$ and temperature difference $\Delta T=15 \mathrm{~K}$ ). For a given windspeed, the vortex is enhanced with increasing temperature.

Figure 5b shows an example of flow streamlines produced for a single vortex regime with a mixed convection case for a canyon with $H / W=1.0$ (windward canyon wall heated, low wind speed $U=2.5 \mathrm{~m} / \mathrm{s}$ and temperature difference $\Delta T=4 \mathrm{~K}$ ). The vortex intensity decreases slightly with increasing temperature due to the opposing forces of buoyancy and forced convection. When the windward wall surface temperature is greater than the air temperature, the air close to the surface is heated, creating an upward buoyancy flux that opposes the direction of bulk entrainment of air at the windward side. Due to the upward movement close to the heated wall, at steady state, the windward bottom vortex is enhanced, with one main clockwise vortex.

At higher buoyancy levels, a change in flow regimes from a single-vortex skimming flow regime to a dual-vortex skimming flow regime was observed. Figure $5 \mathrm{c}$ shows a dual 


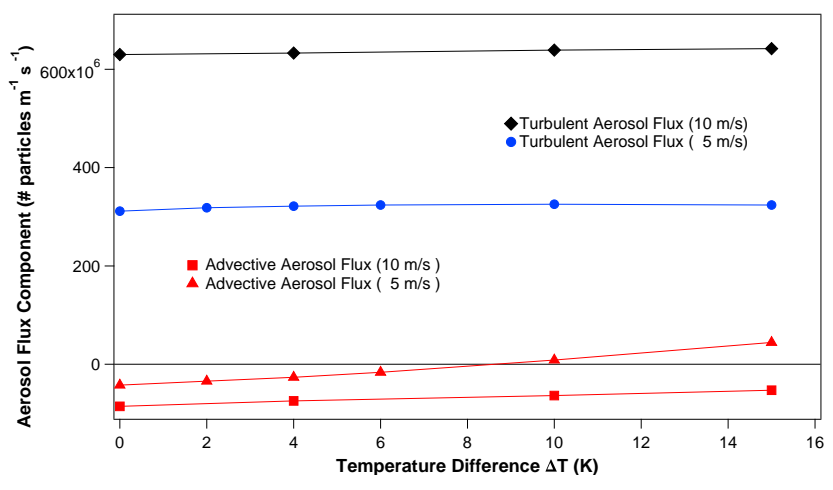

Fig. 12. Aerosol flux components $\left(F_{t}\right.$ and $\left.F_{a}\right)$ vs. Wall Temperature Difference $\Delta T(\mathrm{~K})$ at leeward wall, for $U=5 \mathrm{~m} / \mathrm{s}$ and $10 \mathrm{~m} / \mathrm{s}$.

vortex flow when $\Delta T$ is increased to $15 \mathrm{~K}$ when $U=2.5 \mathrm{~m} / \mathrm{s}$. This flow regime transition takes place when $\Delta T=10 \mathrm{~K}$ at $U=2.5 \mathrm{~m} / \mathrm{s}\left(R_{i}=0.54\right)$. As the transition occurs, the upward movement close to the heated wall splits the main clockwise vortex into two. The upper vortex is weakened as buoyancy is further enhanced.

\subsubsection{Mixed convection: leeward heated wall}

When the leeward wall is heated, the air close to the wall moves upwards, reinforcing the existing main vortex, Fig. 5a. No change in flow regime is observed for all extents of mixed convection considered. Figure 11 summarises the influence of buoyancy on both $F_{a}$ and $F_{t}$ for $U=2.5 \mathrm{~m} / \mathrm{s}$ for increased heating of the leeward canyon wall (temperature difference $\Delta T)$ and Fig. 12 for cases $U=5$ and $10 \mathrm{~m} / \mathrm{s}$ respectively.

For all cases, it was found that $F_{t}$ is positive (net loss of UFP) at the roof level of the canyon and relatively insensitive to $\Delta T . F_{t}$ is a function of the vertical UFP concentration gradient and turbulent diffusivity $\left(K_{\chi}\right)$. Again looking at the variation along the horizontal canyon axis at roof level, for all cases the increase in temperature enhances the concentration gradient at the leeward side of the canyon. Although this should augment the $F_{t}$, the steady state dispersion pattern of the eddy viscosity is such that the mean eddy viscosity along the horizontal axis considered does not vary significantly. This explains the relative stability of $F_{t}$ across different extents of buoyancy.

In contrast, $F_{a}$ was found to be a strong function of $\Delta T$ and a positive linear relationship exists for all extents of mixed convection. When forced convection is dominant the net effect of $F_{a}$ is negative, with re-entrainment of UFP. However, beyond a threshold level of natural convection (when $R_{i}>0.1$ ), $F_{a}$ is positive and would increase linearly, eventually reaching a similar order of magnitude as $F_{t}$ when $R_{i}>\sim 0.43$. Along the horizontal axis, $f_{a}$ is positive at the leeward side and negative at the windward side. At higher buoyancy, the enhancement of $f_{a}$ at the leeward side is significant (Fig. 13), leading to a positive value of $F_{a}$.

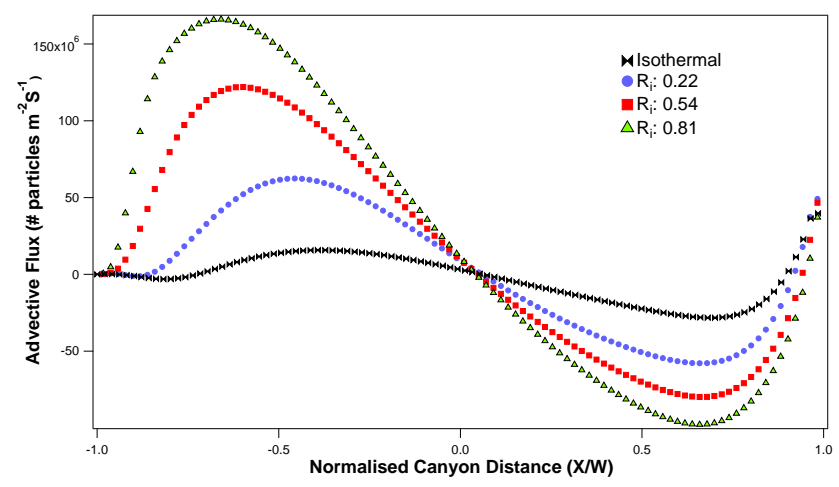

Fig. 13. Advective aerosol flux $\left(f_{a}\right)$ vs. $X / W$ at different extents of mixed convection (leeward wall) and isothermal case.

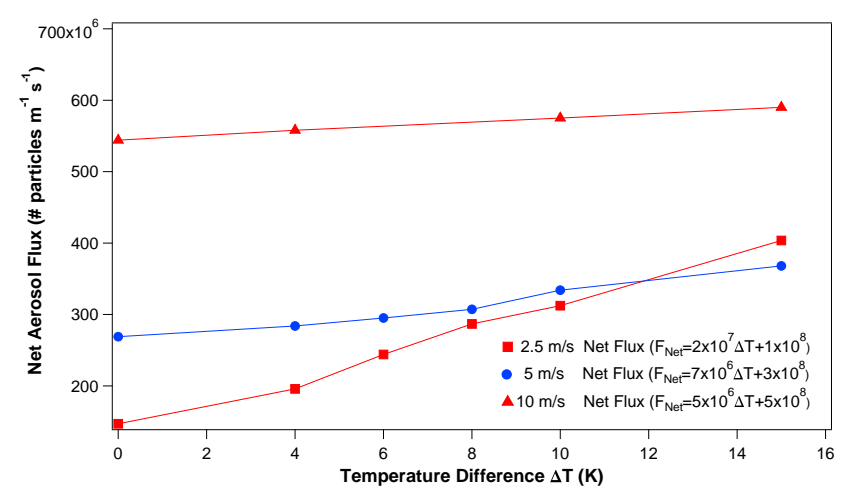

Fig. 14. Net aerosol flux, $F_{\text {Net }, \chi}$, vs. temperature difference, $\Delta T$ (K) at leeward wall.

Net flux, $F_{\text {Net, } \chi}$ shows a positive linear relation with increasing buoyancy, Fig. 14. However, the rate of increase in flux is dependent on the extent of forced convection $(U)$, as shown in the figure. When the temperature difference is $\sim 15$ $\mathrm{K}, F_{\mathrm{Net}, \chi}$ is greater at $2.5 \mathrm{~m} / \mathrm{s}$ than at $5 \mathrm{~m} / \mathrm{s}$. This is due to the dominance of $F_{a}$ due to natural convection at lower $U$. Normalising the increase in flux, $\Delta F$, by the original isothermal flux, $F_{0}$, we can attempt a relationship between the increase in flux with $R_{i}$, i.e.

$$
\left(\frac{\Delta F}{F_{0}}\right)=a R_{i}=a\left(\frac{g H \Delta T}{U^{2} T_{0}}\right)
$$

As expected, a strong positive linear relationship $\left(r^{2}=0.99\right)$ between $R_{i}$ and the enhancement in UFP flux, $\left(\Delta \mathrm{F} / \mathrm{F}_{0}\right)$, due to increasing buoyancy, was found which applies for symmetrical canyons and leeward heated walls with $0<R_{i}<0.81$, Fig. 15. This relationship is a first step towards a simple parameterisation of the UFP flux with differing extents of mixed convection, but simple extension to other heated canyon patterns is not as straightforward as will be shown in Sect. 3.3.3. 


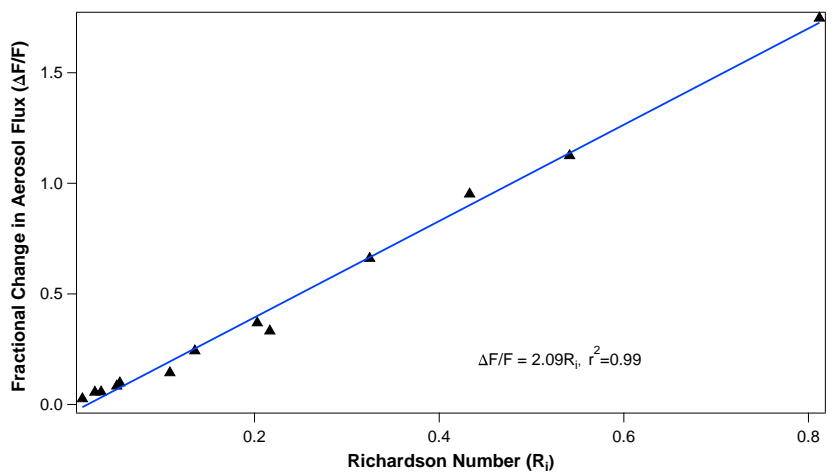

Fig. 15. Enhancement of net aerosol flux $\left(\Delta F / F_{0}\right)$ vs. Richardson Number, $R_{i}$, for the leeward heated wall case.

\subsubsection{Mixed convection: windward heated wall (single-vortex skimming flow regime)}

It was found that the contribution by $F_{t}$ is positive (net loss of UFP from canyon) at the roof level of the canyon for all cases, whilst that for $F_{a}$ is negative. It was also observed that both flux components are relatively insensitive to the temperature difference, although they gently decrease in magnitude with increasing temperature (in spite of the increase in mean turbulent diffusivity), as there is an overall decrease in vertical concentration gradient along the horizontal axis at roof level due to the slightly weakened vortex.

Unlike the leeward case, $F_{a}$ is relatively stable at the range of mixed convection considered (deviating by no more than $10 \%$ ). This is because the main vortex and upper leeward minor vortex is maintained. The main vortex is maintained with no change in the location of its centre, the decrease in vertical advection at the windward side is balanced by an increased vertical advection at the leeward side. Therefore, the effect on $F_{\text {Net, } \chi}$ is not as evident, unlike the leeward wall heated case. As $F_{a}$ is typically an order of magnitude lower than $F_{t}$, the escape of UFP is driven mainly by turbulent flux. This implies that the influence on the UFP flux will not be a directly linear function with respect to $R_{i}$ as was observed for the leeward heated wall case. The fraction of decrease is smaller than the fraction of increase of the leeward heated case ( $<10 \%$ decrease for $R_{i}$ up to 0.20 ) for a given Richardson number, as illustrated in Fig. 16.

\subsubsection{Mixed convection: windward heated wall (dual-vortex skimming flow regime)}

After the transition to a dual-vortex flow regime, although the direction of $F_{t}$ and $F_{a}$ remains the same, the turbulent flux decreases by an order of magnitude (compared to that of advection), Fig. 17. At transition, the effect on the vertical advection is minimal, but a decrease in the amount of UFP re-entrained into the canyon is observed. This is because the flux at that level is due to the upper circulatory vortex, driven

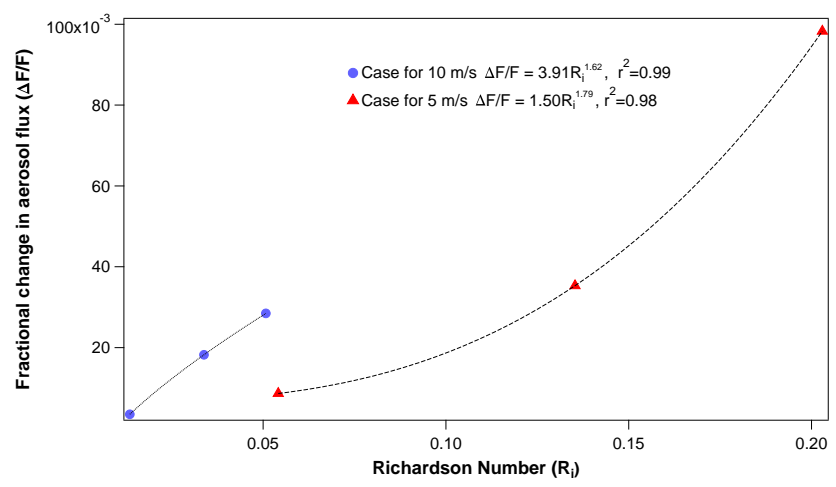

Fig. 16. Fractional change in net aerosol flux, $\Delta F / F_{0}$, vs. Richardson Number, $R_{i}$, for the windward heated wall case and for $U=5 \mathrm{~m} / \mathrm{s}$ and $10 \mathrm{~m} / \mathrm{s}$.

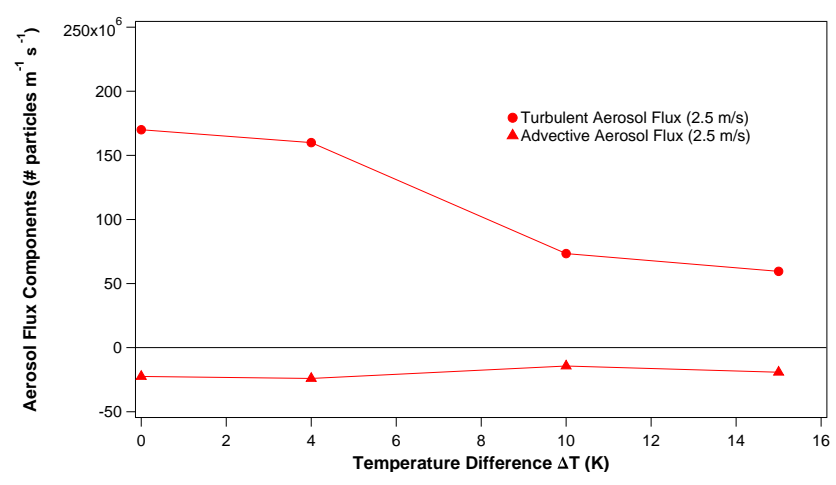

Fig. 17. Aerosol flux components, $F_{t}$ and $F_{a}$ vs. temperature difference, $\Delta T(\mathrm{~K})$ at windward wall for $U=2.5 \mathrm{~m} / \mathrm{s}$.

by forced convection and of relatively lower concentration of UFP while the lower vortex circulates the region of higher UFP concentration.

For windward heated wall cases, flux values at low values of $R_{i}$ were found to be relatively stable until a change of flow regime occurred, when flux values decreased by an order of magnitude, Fig. 18. This result is useful for developing parameterisations for windward heated walls.

\subsubsection{Mixed convection: heat flux}

The influence of heat flux, $F_{\mathrm{Net}, T}$ on UFP flux, $F_{\mathrm{Net}, \chi}$ from the street canyon was investigated along the width of the canyon at roof level. It is expected that $F_{\mathrm{Net}, \chi}$ will increase with enhanced $F_{\text {Net, } T}$ out of the canyon (and vice versa) due to the increase in the vertical velocity arising from natural convection. However, the relationship between heat flux and net UFP flux from the canyon would not be straightforward as will be demonstrated.

Figures 19 and 20 show the relationship between heat and UFP fluxes for the leeward and windward heated canyon walls respectively. UFP flux is a relatively weaker function of heat flux, $F_{\mathrm{Net}, T}$, compared with wind speed. For all cases, 


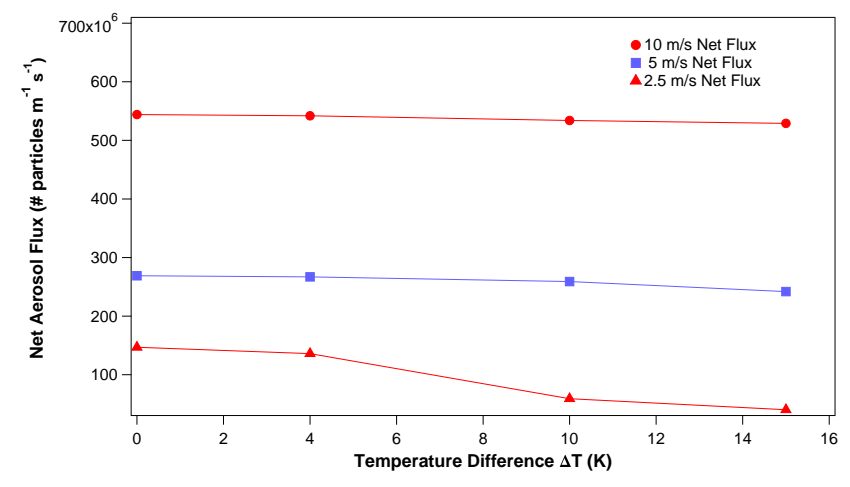

Fig. 18. Net aerosol flux, $F_{\text {Net, } \chi}$, vs. temperature difference, $\Delta T$ (K) at windward wall for $U=2.5,5.0$ and $10.0 \mathrm{~m} / \mathrm{s}$.

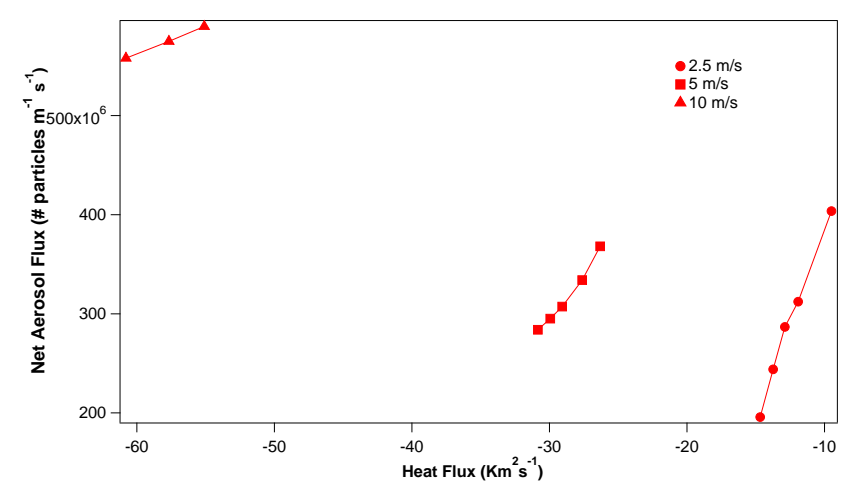

Fig. 19. Net aerosol flux, $F_{\mathrm{Net}, \chi}$, versus canyon top local heat flux, $H,\left(\mathrm{Km}^{2} / \mathrm{s}\right)$ for the leeward heated wall case and for $U=2.5,5.0$ and $10.0 \mathrm{~m} / \mathrm{s}$.

the net heat flux is negative as vertical advective heat flux is more dominant than turbulent heat flux due to a lower vertical temperature gradient at the roof level (compared with UFP gradient). Air at $290 \mathrm{~K}$ is entrained into the canyon at the windward side. Heat transferred from the heated surfaces and the plume $(300 \mathrm{~K})$ is re-circulated into the canyon by the bulk fluid motion and the above canyon mean air temperature remains at $290 \mathrm{~K}$.

In Fig. 19, the enhanced buoyancy due to increasing leeward wall temperature reinforces the vortex and increases the vertical advective flux out of the canyon for both UFP and heat. The sensitivity to enhanced buoyancy increases with decreasing wind speeds.

In Fig. 20, it is observed that for the single vortex regime $(U=5 \mathrm{~m} / \mathrm{s}$ and $10 \mathrm{~m} / \mathrm{s})$, an increase in temperature results in an increase in net heat flux out of the canyon and decrease in net UFP flux out of the canyon. This is due to an increase in turbulent heat flux (due to enhanced vertical temperature gradient) and a lower turbulent UFP flux of a slightly weaker vortex. Nonetheless, these changes are slight. A more pronounced decrease in UFP flux out of the canyon is observed at $2.5 \mathrm{~m} / \mathrm{s}$ when there is a change in flow

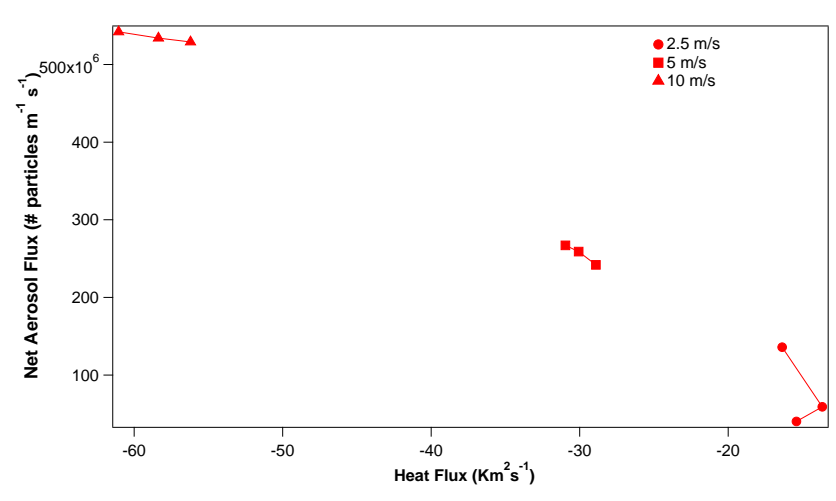

Fig. 20. Net aerosol flux, $F_{\text {Net, } \chi}$, versus canyon top local heat flux, $H,\left(\mathrm{Km}^{2} / \mathrm{s}\right)$ for the windward heated wall case and for $U=2.5,5.0$ and $10.0 \mathrm{~m} / \mathrm{s}$.

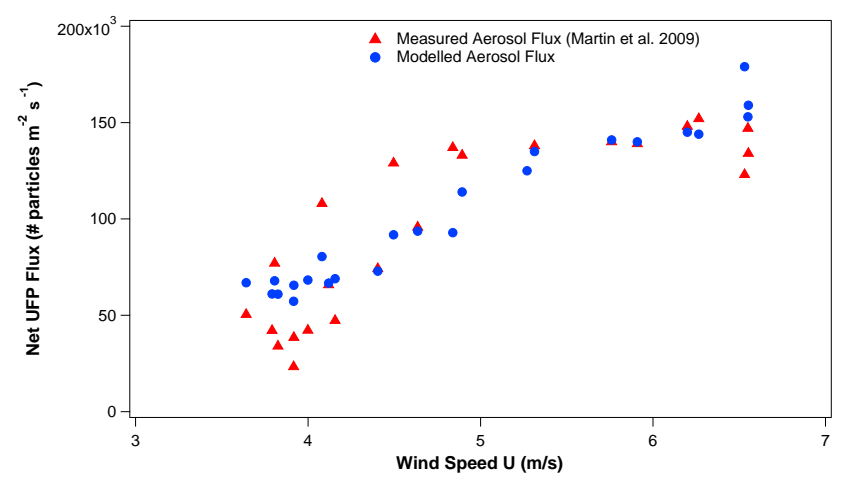

Fig. 21. Modelled UFP aerosol flux compared with observed UFP diurnal averaged measurement of aerosol flux (Data from Martin et al., 2009), $\left(r^{2}=0.65\right)$.

regime. Turbulent UFP flux decreases by an order of magnitude (Sect. 3.3.4) and the vertical advective heat flux into the canyon decreases. With increasing buoyancy, the upper vortex continues to weaken and turbulent UFP flux out of the canyon decreases while vertical advective heat flux into the canyon increases (due to a weaker horizontal velocity and stronger vertical velocity downwards of the weakened upper vortex).

This simple exercise using the first order turbulence model to evaluate fluxes at roof level illustrates the fact that the change in heat flux and UFP flux within canyon scales and different extents of mixed convection do not necessarily correlate positively due to the different factors that drive the flux of both components at different canyon flow conditions. The different processes driving both UFP flux and heat flux will be further investigated using a wider range of turbulence closure methods and boundary conditions. 


\section{Limitations of study}

These studies have only considered an infinitely long canyon, with the wind perpendicular to the canyon axis. They do not consider other wind directions, complex canyon geometry and lateral effects which may influence flux characteristics within actual urban environments.

Though adequate for providing insights into the governing micrometeorological factors to inform field measurement strategies, the use of the standard $k-\varepsilon$ turbulence model approach has its limitations. It assumes that a time averaged, statistically steady description of the turbulent flow may be obtained at long timescales and does not attempt to represent eddies at different scales. It was found to underpredict turbulent diffusion (Walton and Cheng, 2002) and therefore concentration levels may be overpredicted. It also assumes isotropy of turbulence and is not appropriate for modeling cases where anisotropy of turbulence is important, as such impingement (Murakami, 1997). However, the limitations of this model have been found to be less significant for similar symmetrical street canyon cases considered by Dixon and Tomlin (2007).

A wide range of turbulent Schmidt numbers $\left(S c_{t}\right)$ has been proposed ( 0.2 to 1.3$)$ for different flow conditions, depending on the skill of the RANS model in predicting the turbulent eddy viscosity (Tominaga and Stathopoulos, 2007). The $S c_{t}$ was assumed to be 1.0 in all cases, ignoring potential variations to $S c_{t}$ due to varying extents of forced convection and stability. Although this value is slightly higher that what is current used in commercial CFD modelling, (0.7 or 0.9) (Spalding, 1971 and Launder, 1978), it is consistent with $\mathrm{Ku}-$ mar et al. (2009) who considered dispersion of nanoparticles in urban street canyons and is within the range of previously measured values of 0.18 to 1.34 (Flesch, 2002) based on field observations under different atmospheric stability and wind conditions. Nonetheless, although the magnitude of calculated turbulent aerosol particle flux directly depends on the value of $S c_{t}$, qualitative observations of the relative extents of both turbulent and advective fluxes will be unchanged.

These mixed convection model results need to be validated with further field measurements since to date it is believed that numerical simulations overestimate buoyancy effects due to the fact that most full scale field measurements do not show a strong dependence of flow patterns on thermal effects (Solazzo and Britter, 2007; Louka et al., 2002; Oliveria-Panao et at., 2008) although the implications of a change in flow regime for the overall flux pattern will still be valid in spite of the current debate.

There are also quantitative discrepancies between numerical models. The transitional Richardson Number is quantitatively different from results obtained by various groups for a given (or similar) canyon aspect ratio, although it is within the range of most studies. Kovar-Panskus et al. (2002) found the transitional threshold to be of the order of 1 . This is an order of magnitude higher than most other studies. For exam- ple, Sini et al. (1996) estimated the threshold to be $\sim 0.15$, which is lower than the range of values obtained by Mestayer et al. (1995) $(\sim 0.16$ to $\sim 0.5)$ and Oliveria-Panao et al. (2008) ( $\sim 0.25$ to $\sim 0.33)$. It also suggests the sensitivity of the results to mesh configuration and boundary conditions, implying that there is a large uncertainty in the numerically quantified fluxes. Further work is needed to investigate reasons for such variability to better ascertain the transitional Richardson Number for purposes of parameterization into operational models.

\section{Linking aerosol dispersion in urban canyons to neighbourhood-scale emissions}

Here we will attempt a first step towards reconciling tower micrometeorological aerosol turbulent flux (represented as $F_{\text {net }}$ ) measurements made above urban surfaces with the underlying source processes at the street canyon scale. The question that must be considered is whether micrometeorological tower flux measurements above cities can be useful in describing the net ventilation behaviour of canyons with respect to aerosol fluxes.

There have been several field studies of neighbourhoodscale aerosol emissions recently, Dorsey et al. (2002); Martensson et al. (2006); Martin et al. (2009). These have attempted to investigate the relationship between $F_{\text {net }}$ as a function of source parameters such as traffic activity, $T A$, and meteorological factors including wind speed, turbulence, atmospheric stability/and or sensible heat flux. In most cases the studies found strong positive correlation between $F_{\text {net }}$ and some of these factors (generally they show a weak dependence with traditional atmospheric stability parameters).

Dorsey et al. (2002) proposed a parameterisation of the form $F_{\text {net }}=A e^{1.6 T A}$, where A is a constant, $T A$ is the traffic activity (vehicles/s) and $F_{\text {net }}$ is (\#particles $/ \mathrm{cm}^{2} / \mathrm{s}$ ). They also derived a relationship between $F_{\text {net }}$ in terms of the atmospheric stability parameter, $\zeta=-\left(z_{m}-d\right) / L$ (where $z_{m}$ is the measurement height, $d$ the urban canopy zero plane displacement and $L$ the Monin-Obukov length which parameterises the buoyancy to shear driven scales of motion, and is given by $L=-\frac{u_{*}^{3}}{k\left(g_{/} T_{0}\right)\left(H_{0} / \rho C_{p}\right)}$, see Monin and Obukov (1954), where $T_{o}$ and $H_{o}$ are the surface temperature and sensible heat fluxes respectively, $\mathrm{k}=0.4$, von Karman's constant, $C_{p}$ is the specific heat capacity for air, $\mathrm{g}$ the acceleration due to gravity). However the parameterisation was only effective for moderate to strongly unstable cases.

The study by Martensson et al. (2006) proposed a similar approach but used friction speed only; $F_{\mathrm{Net}}=$ $E F_{f m} T A\left(u_{*} / \overline{u_{*}}\right)^{0.4}+f_{0}$, where $E F$ is an emission factor (vehicle/km - in their case they employed a mixed fleet emission factor), $T A$ (in their case) is the traffic activity per unit area and time (vehicle $\mathrm{km} / \mathrm{m}^{2} / \mathrm{s}$ ), $\overline{u_{*}}$ is the average friction 
velocity and $f_{o}$ is the contribution to UFP flux due to nontraffic related sources (and would be negative for losses by deposition).

Martin et al. (2009) using data from the REPARTEE and CityFlux studies proposed that; $F_{\mathrm{Net}}=D\left(a u_{*}+b H\right)+$ $c T A+f_{0}$, where $a, b$ and $c$ are city specific emission factor constants related to friction speed, sensible heat flux and traffic activity respectively, and $D$ is a constant. $T A$, in their case, is the traffic activity (vehicles/s) measured either at a random point or points within or at locations thought to be representative of the measured flux footprint. The reported correlations with $T A$ and $u_{*}$ as expected were the strongest whilst that with sensible heat, $H$ was the weakest. For relatively unstable conditions a positive correlation was found between the sensible heat flux and particle number flux but correlation with local stability, $\zeta$, was poor (also reported by Dorsey et al., 2002).

It is naturally hypothesised that if above canyon micrometeorological aerosol flux measurements are taken above an extensive "uniform homogeneous network" of street canyons, then coupling exists between the flux at some reference height above that network and the average net flux expected at the street canyon top ("urban surface" emissions) in a simple manner. The measured flux at the reference height is usually ascribed a micrometeorological "flux footprint" (e.g. Schuepp et al., 1990) encompassing a surface that conforms to the above definition so that the net flux measured is an aggregation of multiple canyon "aerosol plumes". Here we will compare the flux observations reported by Martin et al. (2009), with our simple canyon parameterisation model. In this approach we assume the following simplifications as a first approximation:

- Coupling of meteorological factors take place between both scales and the effects of horizontal advection may be ignored;

- A well mixed region between both scales exists;

- The aerosol transformation processes within the urban canopy layer will not influence the characteristics of the UFP aerosol number fluxes; and

- Sinks within the urban canopy have a minimal influence on the aerosol flux characteristics and can be ignored.

\subsection{Results: linking aerosol emission fluxes}

If these assumptions are valid, the measured turbulent flux above the urban roughness sublayer and modelled fluxes within canyon scales will relate in a similar way to meteorological factors. MATLAB ${ }^{\circledR}$ routines were used via nonlinear curve fitting to relate the measured diurnal averaged aerosol fluxes (Martin et al., 2009) to the parameterisation (Eq. 5.0), assuming $u_{*}$ is directly proportional to $T I$ and that the contribution of vertical advective flux may be neglected. The measured vertical turbulent fluxes, $\overline{\left(w^{\prime} \chi^{\prime}\right)}$, were obtained using the 3-D-eddy covariance approach, and are rotationally corrected to the locally measured mean horizontal wind component so that the mean vertical velocity component, $\bar{w}$, is zero (e.g. Foken 2006). The following parameterization was recovered: $F_{\mathrm{Net}}=\exp \left(9.92+1.12 \times 10^{-6} \ln U+\right.$ $2.80 T I$ ). We compared a typical parameterisation prediction with the measurement data which resulted in a relatively poor correlation $\left(r^{2}=0.65\right)$ (Fig. 21). The parameterisation was found to under-predict fluxes at low wind speeds and over-predict fluxes at higher wind speeds although general trends were captured. Therefore, although this parameterisation was developed based on idealised conditions within canyon scales, it may be applied as a first approximation for real atmospheric flow conditions within larger scales. This result also suggests that $F_{\text {Net }}$ is a stronger function of wind speed and friction velocity than heat flux/ stability (at least for the city environments considered here) and is consistent with Sect. 3.3.5 as well as the observations although more work is required to refine this.

\subsection{Discussion: linking aerosol emission fluxes}

The less than optimal correlation could be due to a number of reasons. The parameterization does not account for other factors influencing aerosol flux behaviour: heat flux and stability. Such incorporation is challenging due to the complex interaction between natural convection and forced convection. Variability of the source emissions (TA) within the measurement footprints, and the assumption of constant source strength of this parameterisation can also explain the less than optimal correlation. The observational datasets used here were diurnal averages encompassing different meteorological conditions which may be biased towards particular sectors of the city in terms of surface morphology and surface heating which may be different from the flow patterns used to develop the parameterisation. More work should be undertaken to extend the paramterisation for a greater range of flow conditions and surface conditions to improve the results.

The role of aerosol processes between both scales is uncertain. The crux of our simplification and the underlying assumption is that transport lifetimes are shorter than that of aerosol processes which modify particle number. However, we do not rule out the possibility of chemical transformation within urban environments. It has been suggested that dispersion processes alone could not account for the dynamics of nanoparticles within urban areas (Dall'Osto et al., 2009). It is plausible that a significant level of sub-saturation due to the dilution of partial pressure of semi-volatile content during transport between both scales leads to evaporation. How aerosol processes may be included in the parameterization is a topic of further study. 


\section{Summary}

A systematic study was performed to assess the sensitivity of aerosol fluxes at the roof level of idealized canyons to a range of flow conditions. For all "isothermal cases" considered, turbulent flux dominates and is strongly related to wind speed (linear) and turbulent intensities (exponential). A parameterisation characterising this relationship was developed.

Relative to $U$ and $T I$, natural convection has a weaker influence on net aerosol flux. For the leeward heated wall case we observe a reinforcement of the existing vortex and this leads to an enhancement in the aerosol flux. A direct relation between the aerosol flux and Richardson number may be derived. At even higher buoyancy conditions, the effect of leeward side heating is to increase the vertical advective flux to the same order of magnitude as the turbulent flux. As expected, for the case of windward heated canyon walls a slight decrease in overall aerosol flux from canyons is observed at higher Richardson numbers due to the opposing direction of buoyancy to the downward entrainment velocity at the windward side. Under even higher buoyancy conditions the effect of windward wall heating is to alter the flow regime and consequently decrease the turbulent flux component to the same order of magnitude as the vertical advective flux.

The simple parameterization model developed may be used to represent neighbourhood-scale flux emissions, suggesting linkages between urban inertial sublayer and street canyon dynamics. Undoubtedly crude, these comparisons may be used as a starting point for linking mean street level concentrations (but not yet their variability) to those measured above the urban roughness sublayer e.g. on micrometeorological towers. The relationship between aerosol flux and heat flux was found to be weak, possibly due to the complex interactions of mixed convective forces and heterogeneity of urban geometries. Clearly more work is needed to refine these linkages between CFD and tower based studies using improved statistical representations of canyon characteristics within flux footprint approaches.

Acknowledgements. This work was supported by DSO National Laboratories, Singapore. Data was provided by the NERC funded CityFlux grant, NE/B504865/1 and the REPARTEE project, funded by BOC Ltd.

Edited by: W. T. Sturges

\section{References}

Baik, J. J. and Kim, J. J.: A numerical study of flow and pollutant dispersion characteristics in urban street canyons, J. Appl. Meteorol., 38, 1576-1589, 1999.

Baik, J. J. and Kim, J. J.: On the escape of pollutants from urban street canyons, Atmos. Environ., 36, 527-536, 2002.

Baik, J. J. and Kim, J. J.: Effects of inflow turbulence intensity on flow and pollutant dispersion in an urban street canyon, J. Wind Eng. Ind. Aerodynam., 91, 309-329, 2003.

Barlow, J. F. and Belcher, S. E.: A wind tunnel model for quantifying fluxes in the urban boundary layer, Bound.-Lay. Meteorol., 104, 131-150, 2002.

Belcher, S. E.: Mixing and transport in urban areas, Phil. Trans. Roy. Soc., 363, 2947-2968, 2005.

Chan, T. L., Dong, G., Leung, C. W., Cheung, C. S., and Hung, W. T.: Validation of a two-dimensional pollutant dispersion model in an isolated street canyon, Atmos. Environ., 36, 861-872, 2002.

Dall'Osto, M., Harrison, R. M., Thorpe, A., Williams, P. and Coe, H.: Remarkable dynamics of nanoparticle in the urban atmosphere, European Aerosol Conference 2009, Karlsruhe, Germany, Abstract T130A06, 2009.

DePaul, F. T. and Sheih, C. M.: A tracer study of dispersion in an urban street canyon, Atmos. Environ., 19, 555-559, 1985.

Dixon, N. S. and Tomlin A. S.: Evaluation of a lagrangian model for predicting concentration fluctuations in urban areas, Proceedings $11^{\text {th }}$ International Conference on Harmonization within Atmospheric Dispersion Modelling for Regulatory Purposes, 2007.

Dorsey, J. R., Nemitz, E., Gallagher, M. W., Fowler, D., Williams, P. I., Bower, K. N., and Beswick, K. M.: Direct measurements and parameterisation of aerosol flux, concentration and emission velocity above a city, Atmos. Environ., 36, 791-800, 2002.

Ferziger, J. H. and Peric, M.: Computational Methods for Fluid Dynamics (3rd Edition), Springer-Verlag, Berlin, Heidelberg, Germany, 423 pp., 2001.

Flesch, T. K.: Turbulent Schmidt number from a tracer experiment, Agr. Forest Meteorol., 111, 299-307, 2002.

Foken, T.: Micrometeorology, Springer-Verlag, Berlin, Berlin, 306 pp., 2006.

Gallis, M. A., Torczynski, J. R., and Rader, D. J.: Nonzeroconcentration boundary condition for advection-diffusion aerosol-transport modeling, Aerosol Sci. Technol., 42, 829-831, 2008.

Hunter, L. J., Johnson, G. T., and Watson, I. D.: An investigation of three dimensional characteristics of flow regimes within the urban canyon, Atmos. Environ., 26B, 425-432, 1992.

Jeong, S. J. and Andrew, M. J.: Application of the k-epsilon turbulence model to high reynolds number skimming flow field of an urban street canyon, Atmos. Environ., 36, 1137-1145, 2002.

Johnson, J. T. and Hunter, L. J.: Some insights into typical urban canyon airflows, Atmos. Environ., 33, 3991-3999, 1999.

Ketzel, K., Louka, P., Sahm, P., Guilloteau, E., and Sini, J.-F.: The use of computational fluid dynamics in modelling air quality in street canyons in Optimisation of Modelling Methods for Traffic Pollution in Street, Final Report for EU Trapos Project, edited by: Berkowicz, R., Britter, R., and Sabatino, S. D., Chapter 2.2, 86-93, available online at: http://www2.dmu.dk/AtmosphericEnvironment/trapos/ TheBook/TRAPOS_Book.pdf, 2004.

Kittelson, D. B.: Engines and nanoparticles: a review, J. Aerosol 
Sci., 29, 575-588, 1998.

Kovar-Panskus, A., Moulinneuf, L., Savory, E., Abdelqari, A., Sini, J.-F., Rosant, J.-M., Robins, A., and Toy, N.: A wind tunnel investigation of the influences of solar-induced wall heating on the flow regime within a simulated urban street canyon, Water Air Soil Poll., 2, 555-571, 2002.

Kumar, P., Garmory, A., Ketzel, M., Berkowicz, R., and Britter, R.: Comparative study of measured and modelled number concentrations of nanoparticles in an urban street canyon, Atmos. Environ., 43, 949-958, 2009.

Launder, B. E. and Spalding, D. B.: The numerical computation of turbulent flows, Comput. Meth. Appl. Mech. Eng., 3, 269-289, 1974.

Launder, B. E.: Heat and Mass Transport, in: P. Bradshaw (Editor): Topics in Applied Physics, Turbulence,, Springer, Berlin, Germnay, 12, 231-287, 1978.

Longley, I. D.: Corrections to a description of turbulence in a trafficked street canyon in Manchester, Atmos. Environ., 38, 45894592, 2004.

Louka, P., Vachon, G., Sini, J. F., Mestayer, P. G., and Rosant, J. M.: Thermal effects on the airflow in a street canyon- Nantes'99 Experimental results and model simulations, Water Air Soil Poll. Focus, 2, 351-364, 2002.

Mårtensson, E. M., Nilsson, E. D., Buzorius, G., and Johansson, C.: Eddy covariance measurements and parameterisation of traffic related particle emissions in an urban environment, Atmos. Chem. Phys., 6, 769-785, 2006, http://www.atmos-chem-phys.net/6/769/2006/.

Martin, C., Longley, I. D., Dorsey, J. R., Thomas, R. M., Gallagher, M. W., and Nemitz, E.: Ultrafine particle fluxes above four major European cities, Atmos. Environ., 43, 4714-4721, 2009.

Mestayer, P. G., Sini, J.-F. and Jobert, M.: Simulation of the Wall Temperature Influence on Flows and Dispersion within Street Canyons, 3rd International Conference on Air Pollution, Porto Carras, Greece, Computational Mechanics Publication, 1, 109116, 1995.

Meroney, R. N., Pavageau, M., Rafailidis, S., and Schatzmann, M.: Study of line source characteristics for 2-D physical modelling of pollutant dispersion in street canyons, J. Wind Eng. Ind. Aerodynam., 62, 37-56, 1996.

Monin, A. S. and Obukov, A. M.: Basic laws of turbulent mixing in the ground layer of the atmosphere, Acad. Nauk SSSR Tr. Geofiz., Inst., 245, 163-187, 1954.

Murakami, S.: Current Status and future trends in computational wind engineering, J. Wind Eng. Ind. Aerodynam., 67-68, 3-34, 1997.

Murena, F., Favale, G., Vardoulakis, S., and Solazzo, E.: Modelling dispersion of traffic pollution in a deep street canyon: Application of CFD and operational models, Atmos. Environ., 43, 23032311, 2009.
Nakamura Y. and Oke T. R.: Wind, temperature and stability conditions in an east-west oriented urban canyon, Atmos. Environ., 22, 2691-2700, 1988.

Oliveria Panão, M. J. N., Gonçlaves, H. J. P., and Ferrão, P. M. C.: Numerical analysis of the street canyon thermal conductance to improve urban design and climate, Build. Environ., 44, 177-187, 2008.

Patankar, S. V.: Numerical Heat Transfer and Fluid Flow, McGrawHill, NY, USA, 197 pp., 1980.

Savory, E., Rotach, M. W., Chauver, C., Guilloteau, E., KasterKlein, P., Kovar-Panskus, A., Louka, P., Sahm, P., and Castelli, S. T.: Street architecture and air quality in Optimisation of Modelling Methods for Traffic Pollution in Street, Final Report for EU Trapos Project, edited by: Berkowicz, R., Britter, R., and Sabatino, S. D., Chapter 1.1, 8-24, available online at: http://www2.dmu.dk/AtmosphericEnvironment/trapos/ TheBook/TRAPOS_Book.pdf, 2004.

Schuepp, P. H., Leclerc, M. Y., MacPherson, J. I., and Desjardins, R. L.: Footprint Prediction of Scalar Fluxes. From Analytical Solutions of the Diffusion Equation, Bound.-Lay. Meteorol., 50, 355-373, 1990.

Sini, J.-F., Anquetin, S., and Mestayer, P. G.: Pollutant dispersion and thermal effects in urban street canyon, Atmos. Environ., 30, 2659-2677, 1996.

Solazzo, E. and Britter, R. E.: Transfer processes in a simulated urban street canyon. Bound.-Lay. Meteorol., 124, 43-60, 2007.

Spalding, D. B.: Concentration fluctuations in a round turbulent free jet, J. Chem. Eng. Sci., 26, 95-107, 1971.

Tominaga, Y. and Stathopoulos, T.: Turbulent Schmidt numbers for CFD analysis with various types of flowfield, Atmos. Environ., 41, 8091-8099, 2007.

Versteeg, H. K. and Malalasekera, W: An Introduction to CFD. The Finite Volume Method, 2nd edn., Longman, London, UK, 2007.

Walton, A. and Cheng A. Y. S.: Large-eddy simulation of pollution dispersion in an urban street canyon-Part II: idealised canyon simulation, Atmos. Environ., 36, 3615-3627, 2002.

Whitby, E. R., McMurry, P. H., Shankar, U., and Binkowski, F. S.: Modal Aerosol Dynamics Modeling, Rep. 600/3-91/020, Atmospheric Research and Exposure Assessment Laboratory, US Environmental Protection Agency, Research Triangle Park, NC, available as NTIS PB911617291AS from National Technical Information Service, Springfield, VA, USA, 1991. 